\title{
A Bandwidth-saving Optimization for MPI Broadcast Collective Operation
}

\author{
Huan Zhou \\ High Performance Computing Center Stuttgart (HLRS) \\ University of Stuttgart, Germany
}

\author{
Christoph Niethammer \\ High Performance Computing Center Stuttgart (HLRS) \\ University of Stuttgart, Germany
}

\begin{abstract}
The efficiency and scalability of MPI collective operations, in particular the broadcast operation, plays an integral part in high performance computing applications. MPICH, as one of the contemporary widely-used MPI software stacks, implements the broadcast operation based on point-to-point operation. Depending on the parameters, such as message size and process count, the library chooses to use different algorithms, as for instance binomial dissemination, recursive-doubling exchange or ring all-to-all broadcast (allgather). However, the existing broadcast design in latest release of MPICH does not provide good performance for large messages (lmsg) or medium messages with non-power-of-two process counts (mmsg-npof2) due to the inner suboptimal ring allgather algorithm. In this paper, based on the native broadcast design in MPICH, we propose a tuned broadcast approach with bandwidth-saving in mind catering to the case of lmsg and mmsg-npof 2 . Several comparisons of the native and tuned broadcast designs are made for different data sizes and program sizes on Cray XC40 cluster. The results show that the performance of the tuned broadcast design can get improved by a range from $2 \%$ to $54 \%$ for $l m s g$ and mmsg-npof 2 in terms of user-level testing.
\end{abstract}

Index Terms-MPICH, Broadcast, Bandwidth-saving

\section{INTRODUCTION}

The message passing interface (MPI) [1] standard provides a flexible and portable environment for developing high performance parallel applications on different platforms. Since the release of the first version of MPI, it has become a very flexible communication layer providing mechanisms for both point-to-point and collective operations.

The MPI standard specifies various types of collective operations [2] such as All-to-All (MPI_Allgather, MPI_Allscatter and MPI_Alltoall), All-to-One (MPI_Gather and MPI_Reduce) and One-to-All (dissemination: MPI_Bcast and MPI_Scatter). Many scientific applications use collective communications to synchronize or exchange data.

Noticeably, collective communication is a critical and also frequently used component in MPI. In particular, MPI broadcast, as the typical One-to-All interface, is an essential interface widely used in many scientific fields, for instance, matrix multiplication, basic linear algebra [3] and so on. Besides, a profiling study [4] shows that the efficiency of MPI broadcast operations can have a remarkable impact on the overall LS-

\author{
Vladimir Marjanovic \\ High Performance Computing Center Stuttgart (HLRS) \\ University of Stuttgart, Germany \\ José Gracia \\ High Performance Computing Center Stuttgart (HLRS) \\ University of Stuttgart, Germany
}

DYNA [4] performance. Therefore, the MPI implementors are willing to put great efforts on the optimization of MPI broadcast implementation.

MPICH [5] is a high-performance, freely-available and portable implementation of the MPI and MPICH3 supports the latest version of the MPI standard - MPI-3. In addition, A vast majority of other MPI implementations, including IBM MPI, Intel MPI, Cray MPI, OSU MVAPICH/MVAPICH2 and so forth, are derived from MPICH. According to the new Top500 Supercomputer report [6], of the top 10 Supercomputers as of November 2014, 90\% are exclusively using MPICH or its derivatives.

Multi-core processor [7] emerges to speed up the computation capability of processor through performing the workload among multiple cores concurrently. With the advantage of multi-core processor, it has been commonly deployed in the nowadays computation clusters. Accordingly, we can basically exploit two communication levels - intra-node and internode [8] on multi-core clusters to analyze the broadcast algorithm in MPICH3.

MPICH has already highly tuned the implementation of broadcast operation in a way of using multiple algorithms based on the message size and process count [9] and such implementation design is still employed in MPICH3 [10]. However, there is still considerable room left at optimizing broadcast especially when transferring the long messages (lmsg), or medium messages but with non-power-of-two process counts (mmsg-npof2). The occurrence of non-power-oftwo processes can be due to explicit user request at joblaunching, particularly on systems where the core count per node is already non-power-of-two, or due to splitting on the communicator in the applications.

For lmsg, MPICH3 adopts a scatter-ring-allgather approach, where the source data to be broadcast is first divided up and scattered among the processes following a binomial tree from root. The scattered data are then collected back to all processes as a ring allgather operation. For mmsgnpof 2, the implementation is of multi-core awareness, where we first perform an intra-node broadcast on the node of root following a binomial tree, then perform the inter-node 
broadcast by using the scatter-ring-allgather, finally perform the intra-node broadcast on all nodes except for the node of root again following a binomial tree. However, the suboptimal ring allgather operation potentially involves verbose data transmissions which actually can be avoided. Further, for lmsg, it is to be noted that we need put considerable emphasis on the usage of bandwidth, which implies that the number of useless data transmissions should be minimized aiming to save bandwidth use. This is an interesting subject that is however easily overlooked by most active MPI researchers.

Therefore, in this paper, we investigated in-depth the methodology of designing an optimized broadcast algorithm (scatter-ring-allgather) particularly for lmsg and mmsg-npof 2 by tuning the suboptimal ring allgather design. The scalability and portability of the optimized broadcast algorithm can be maintained since such optimization is not bound to particular platform or special features in hardware.

We have implemented the native and tuned scatter-ringallgather algorithms without multi-core awareness on the user level. Hence we can compare the native and tuned scatterring-allgather algorithms for lmsg and mmsg-npof 2 case from a broader perspective, which allows us to observe their performance difference when the inter-node or intra-node data transmissions are involved. Here on, all references to the user-level implementation of the native and tuned scatterring-allgather algorithms free of multi-core awareness refer to MPI_Bcast_native and MPI_Bcast_opt, respectively. We conduct a series of experiments on Cray XC40 to indicate that the tuned design can improve the bandwidth performance of broadcast operation by a range from $2 \%$ to $54 \%$ for $l m s g$ and mmsg-npof 2 in terms of the user-level testing.

The rest of this paper is organized as follows: In Section [II we discuss related work. In Section III] we provide an overview of the native scatter-ring-allgather algorithm (MPI_Bcast_native). In Section IV] the tuned design of scatter-ring-allgather algorithm (MPI_Bcast_opt) is described and explained with the pseudo-code as well. We evaluate the tuned design and compare it with MPI_Bcast_native in Section V. Conclusion is presented in Section VI

\section{RELATED WORK}

There have been many careful studies about the optimization of broadcast implementation targeting for the specific interconnects. Two papers [11], [12] focus on the InfiniBand clusters with hardware-supported multicast, which can improve the overall performance of broadcast significantly and however are closely dependent of the underlying interconnects. Additionally, A study [13] demonstrates that the broadcast performance can get improved on the Software-Designed network, which is of controllability. The MPI broadcast operations get optimized as a result of the network hardware acceleration for broadcast provided by the Blue Gene/Q, shown in paper [14]. However, those optimized designs will show poor portability when applying them on other networks without multicast support, controllability or dedicated hardware acceleration.

\section{BACKGROUND}

\section{A. Overview of MPI_Bcast_native}

MPI standard specifies that the broadcast operation should disseminate a message from a root process to other processes in a communication group. MPI broadcast is a blocking operation, which means all processes are ready to use the received data after the broadcast operation is successfully returned.

The algorithm that is generally used by MPI_Bcast_native for lms $\mathrm{m}$ and mmsg-npof 2 is the combination of a binomial scatter and a suboptimal ring allgather operation. We assume that there are $P$ processes participating in the broadcast operation. In theory, MPI_Bcast_native first uses a binomial scatter to make process $i$ get the $i$-th block of data from root (Section $\mathrm{I}$ mentioned that the data source should be divided up into $P$ pieces of data block before the scatter operation), then invokes a $(P-1)$-step enclosed-ring allgather operation.

On the one hand, we explain how the scatter operation proceeds following a binomial tree from the root 0 for a powerof-two number of processes -8 processes, shown in Figure 1. The root 0 divides the source data into 8 chunks, where each chunk will be marked as a non-negative number $i$ and supposed to be transmitted to the corresponding process $p i$. In this way the source data in root 0 consists of 8 chunks of data marked with $0,1, \ldots, 7$ sequentially. In the first step, root 0 sends the chunk set of $\{4,5,6,7\}$ to process 4 , then a subtree is spawned, as process $p 4$ be the root. In the following step, we spawn two new sub-trees, as process 2 and 6 be the root respectively. Finally each process is able to get the corresponding data after the third step and a binomial tree spanning 8 processes is completed. Generally all processes can get the corresponding data in $\log _{2} P$ steps for power-oftwo processes. On the other hand, we, take 10 processes for example, describe the generation of a binomial scatter tree for non-power-of-two processes. The scatter path keeps the same as the path in Figure 1 except that an additional branch, as process 8 be the root, is spawned. In this case the entire scatter operation finishes in $\left\lceil\log _{2} P\right\rceil$ steps, shown in Figure 2 According to the above two figures, we can conclude that practically not only does each non-leaf node $p i$ in the binomial scatter tree own its corresponding chunk of data marked with $i$, but it also provides all data chunks for its descendant.

Figure 3 shows us the suboptimal ring allgather operation for 8 processes, process $i$ sends its contribution to process $i+1$ and meantime receives the contribution from process $i-1$ (with wrap-around) in the first step and from the second to seventh step each process $i$ forwards to process $i+1$ the data it received from process $i-1$ during the previous step. The set that is listed above each process indicates all data chunks that it owns in reality after the binomial scatter operation, illustrated in Figure 1 However we can find that this allgather algorithm is carried out in an enclosed ring manner, where each process $p i$ pretends to only own the $i$-th data chunk and thus repeatedly receives the data chunks that already existed in it. This obviously leads to a large amount of useless data trans- 


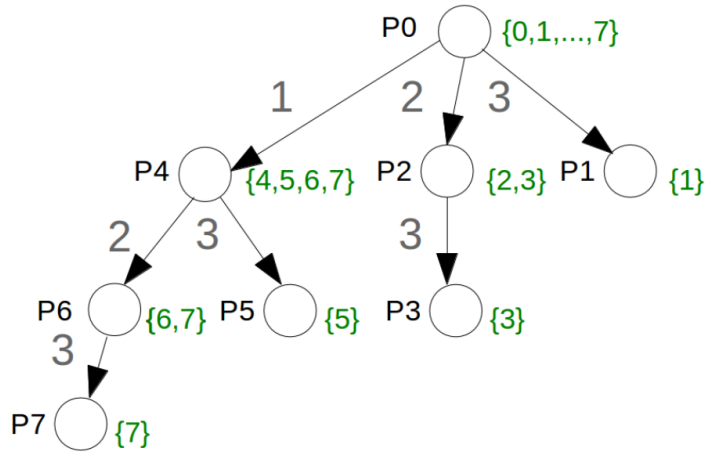

Fig. 1. Schematic for the binomial scatter operation in the case of 8 processes

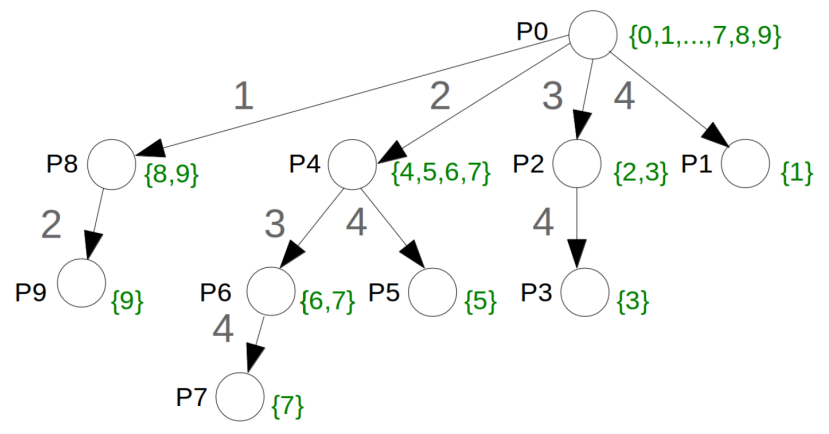

Fig. 2. Schematic for the binomial scatter operation in the case of 10 processes

missions in each step. For the non-power-of-two processes, the algorithm of ring allgather operation goes the same way as that for power-of-two processes. Therefore, generally there are totally data transmissions of $P \times(P-1)$ in this suboptimal ring allgather operation with data transmissions of $P$ at each step.

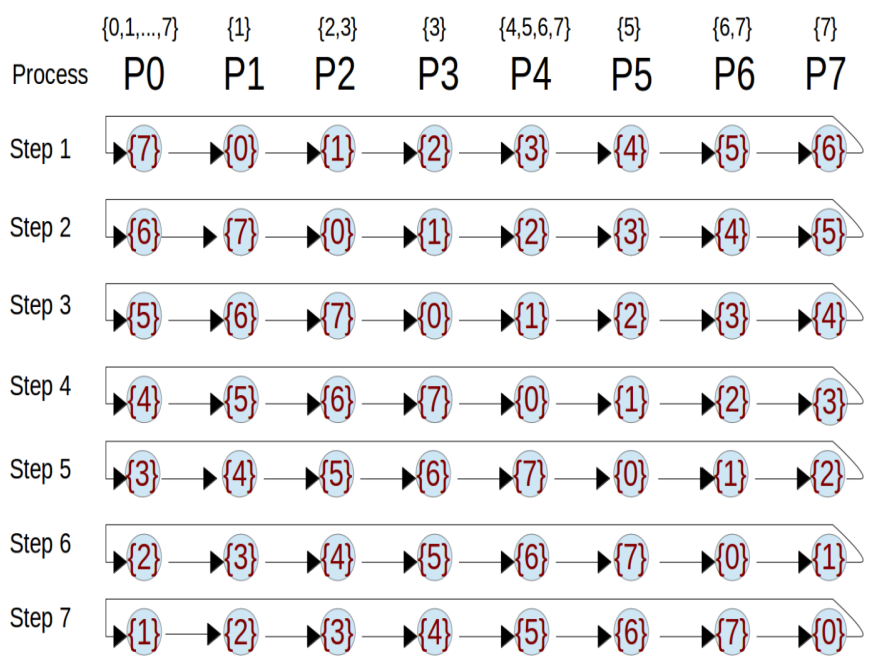

Fig. 3. Enclosed ring for the original allgather algorithm in the case of 8 processes

\section{Designing MPI_Bcast_opt}

In this section, we describe the new broadcast algorithm for lms $g$ and mmsg-npof 2 and its potential advantage over the original algorithm used by MPICH3, which has been elaborated in Section III

We optimize the native broadcast algorithm in MPICH3 by tuning the suboptimal ring allgather operation in a way of letting each process be aware of the actual data chunks that it already owned and skipping the verbose data transfers happening in the original ring allgather operation (see Figure 3). Figure 4 shows the tuned ring allgather algorithm by illustrating the send and receive events that happen in each step with run size of 8 .

Likewise, the set shown in the top row lists all data chunks that a process has already owned after doing the binomial scatter operation. Noticeably, we can see that the new ring allgather algorithm proceeds in a non-enclosed ring manner. Process 0 is the root owning the source data and thus it does not need receive any chunk of data from process 7 . In the first four steps, process 4 gets the data chunks marked with 3,2,1 and 0 from process 3 in sequence, which complete the receive buffer of process 4 together with its existing chunks of data marked with 4,5,6 and 7. Therefore, from the fifth step on process 4 stop receiving any further data chunk from process 3 . by analogy, we can see that process 2 and 6 collect data chunks from process 1 and 5 till the seventh step, where process 2 and 6 already gets all the data chunks that they are lack of. Therefore, each process only receives those missing data chunks, ignores the repeated data chunks and also terminates in $8-1=7$ steps. The number of message transfers in the original ring allgather algorithm is $8 \times(8-1)=56$ for 8 processes, by contrast, the tuned design can reduces it by 12 .

Figure 5 exhibits a more complex scenario, where the tuned ring allgather algorithm performs with 10 processes as an example of non-power-of-two. Process 4 stops receiving data chunks from process 3 after reaching the sixth step since it already gets all missing data chunks marked with 3,2,1,0,9 and 8 sequentially. Additionally, not only process 2 and 6 but also process 8 get the full source data chunks which are broadcast initially by root after eighth step. In this case, the number of message transfers is 75 and reduced by 15 compared with $10 \times(10-1)=90$ brought by the original ring allgather algorithm.

According to Figures 4 and 5, we can deduce that the decrement in the amount of the transferred data will increase as the growing of the process count $P$. Furthermore, by combining Figure 3 and Figure 4 , we can conclude that the tuned ring allgather algorithm reduces the data transmission traffic to an extent using the same steps as the native ring allgather algorithm.

Intuitively, the tuned ring allgather algorithm can speedup the broadcast performance by reducing unnecessary data transmissions. Technically, in the case of intra-node, the point-topoint operation is implemented via memory copying, which is considered to involve the cpu-interference and buffer memory 


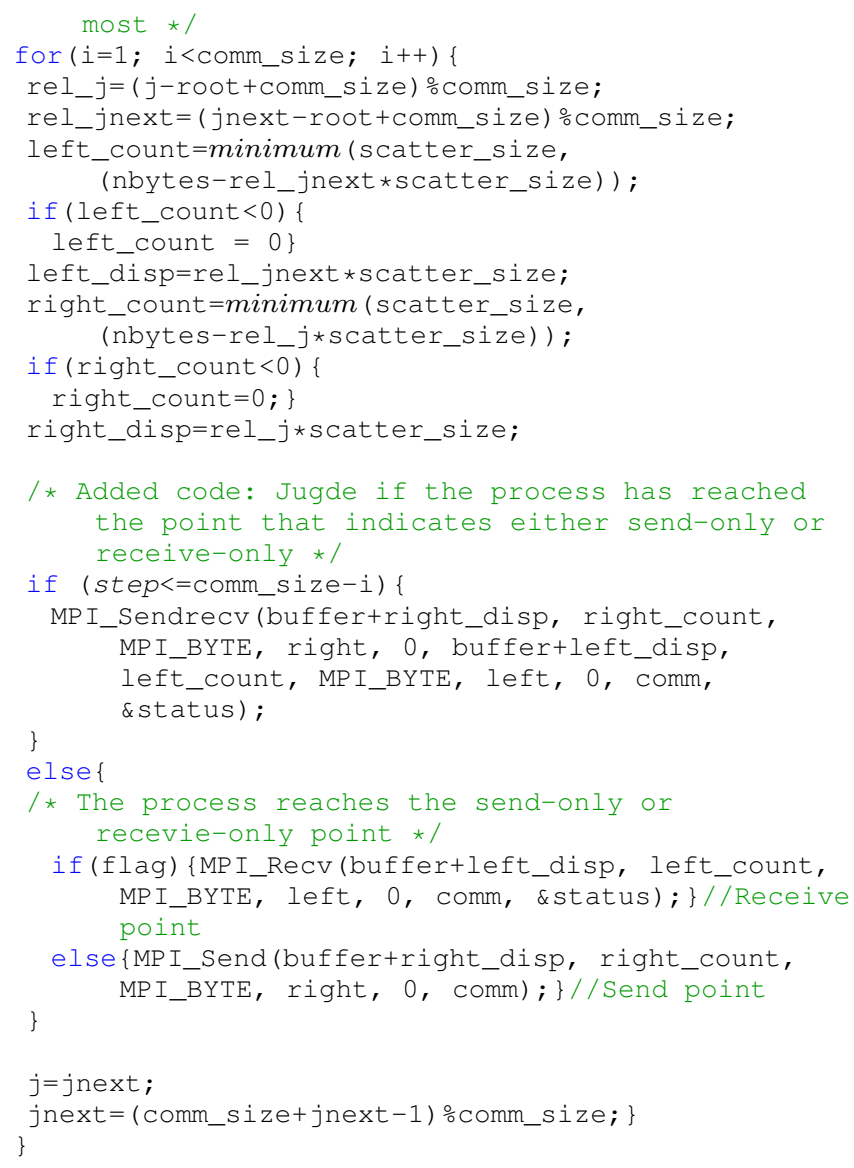

\section{EXPERIMENTAL EVALUATION}

In this section we describe our experiments and give a detailed explanation of the comparison results. We conducted the micro communication benchmarks for broadcast to test its bandwidth on two clusters with the following configures for our tests:

1) Cray XC40, called Hornet: dual Intel Haswell E5$2680 \mathrm{v} 32.5 \mathrm{GHz}$ processors compute node with $128 \mathrm{~GB}$ of main memory, 24 cores per node. The nodes are interconnected through a Cray Aries network using Dragonfly topology.

2) NEC Cluster, called Laki: dual Intel Xeon X5560 2.80 $\mathrm{GHz}$ processors compute node with $8 \mathrm{MB}$ L3 Cache, 8 cores per node. The nodes are interconnected via Infiniband using switched fabric topology.

All processes are synchronized with a MPI barrier before reaching the broadcast interface. We then repeat the broadcast operation for 100 iterations and report the bandwidth. Note, that the bandwidth we present in this section is the rate at which the broadcast messages can be processed, and measured in Megabytes per second $(\mathrm{MB} / \mathrm{s})$. Note, that throughout this paper we use megabytes (MB) and kilobytes (KB) in the base2 sense, i.e., $2^{20}$ and $2^{10}$, respectively.

The message size threshold determined by MPICH3 to switch from short messages to medium messages is 12288 bytes and the message size threshold to switch from medium messages to long messages is 524288 bytes. Thus, we suppose that the long messages should be larger than 524287 in bytes and medium messages should be larger than 12287 and smaller than 524288 in bytes. Our experiments are classified into two types. First, we evaluate the tuned design in the case of long message transmission varying the sizes from 524288 to 30000000 bytes with the number of processes of 16,64 and 256 respectively. Second, we evaluate the tuned design for medium messages and long messages with non-powerof-two processes, as for example 9, 17, 33, 65 and 129 processes. Third, we measure the tuned design for a range of message sizes from 12288 to 2560000 bytes with 129 running processes.

Now we introduce two comparison objects for the two experimental platforms - Hornet and Laki. For Hornet, we implement the native and tuned broadcast algorithms on the user-application level (mentioned in Section I). The compiler we used is Cray compiler. For Laki, we implement the tuned broadcast algorithm on the MPI level, which is altered directly inside the MPI code. Therefore, the compiler we used is the MPICH in-build compiler - mpicc.

In this section, we only present the comparison results on Hornet since the results from both Hornet and Laki basically deliver the same bandwidth performance trend.

\section{A. Long Messages with Power-Of-Two Processes}

In this experiment, we measure the bandwidth performance of MPI_Bcast_native and MPI_Bcast_opt on Hornet over a range of long message sizes with power-of-two processes, as for example 16, 64 and 256 processes. All data transmissions occur within one node when only 16 processes are launched since all the processes are placed among the nodes in a blocked manner by default on Hornet. The comparison results are shown in Figure 6 for Hornet system. The results are explained as follows:

16 processes: As described in Section IV] when the broadcast operation only involve the intra-node data transmission, the tuned allgather design can avoid extra memory copying operations, which can help to save the memory source consumption and alleviate host processing overhead in the MPI_Bcast_opt implementation. The bandwidth performance results for 16 processes are shown in Figure 6[a) We observe an improvement of as high as $12 \%$ for bandwidth as comparison to MPI_bcast_native at $512 \mathrm{~KB}$, The results also show us that the MPI_Bcast_opt consistently outperforms MPI_Bcast_native even for very large messages (beyond 10MB). Additionally, The MPI_Bcast_opt and MPI_Bcast_native report a peak bandwidth of up to 2748 $\mathrm{MB} / \mathrm{s}$ and $2623 \mathrm{MB} / \mathrm{s}$, respectively. We see that the peak bandwidth performance of MPI_Bcast_opt is slightly (10\%) better than that of MPI_Bcast_native.

64 processes: When 64 processes are launched on Hornet, not only is the intra-node data transmission involved in the broadcast, but the inter-node data transmission also play a certain role in the broadcast operation. The growing number of outgoing inter-node messages will increase the burden 
of network routing. As can be noted from the Section IV. compared to MPI_Bcast_native, the number of inter-node messages will be reduced to an extent in the MPI_Bcast_opt algorithm, which leads to the improvement of the overall broadcast performance in bandwidth. Further, Figure G[(b) shows the bandwidth results for 64 processes. Comparing with the MPI_Bcast_native we can observe that the bandwidth achieved by the MPI_Bcast_opt can be increased by as high as $41 \%$ for 64 processes on Hornet. The MPI_Bcast_opt performs $13 \%$ better than the MPI_Bcast_native in peak bandwidth.

256 processes: There are more inter-node messages injecting into network for 256 processes than for 64 processes, Therefore, in the case of 256 processes, the performance of the broadcast depends on the network efficiency more than the case of 64 processes and also the inter-node message transmissions form a greater portion of the overall cost of broadcast operation. The results shown in Figure G(c) indicate that MPI_Bcast_opt can yield an improvement of up to $20 \%$ in bandwidth as comparison to MPI_Bcast_native for 256 processes on Hornet. Additionally, compared with MPI_Bcast_native, the MPI_Bcast_opt improves the peak bandwidth by $16 \%$ for 256 processes. Moreover, The curves shown in Figure G(c), Figure $6(\mathrm{~b})$ and Figure $6(\mathrm{a})$ for Hornet suggest that the MPI_Bcast_opt gains the biggest peak bandwidth advantage for 256 processes. The drop in bandwidth performance of MPI_Bcast_opt and MPI_Bcast_ori starts from around $4 \mathrm{MB}$ for 16 processes, is attributed to the limited memory capacity. Likewise, the bandwidth performance shows slow growth as the increase of transfer message sizes for 64 processes. We see that there is a sudden drop at around $3 \mathrm{MB}$ for 256 processes due to cache effects.

\section{B. Medium Messages and Long Messages with Non-Power- Of-Two Processes}

In this experiment, we first test the throughput (here denoted as the broadcast messages per second) speedups of MPI_bcast_opt over MPI_Bcast_native for medium messages (take two critical message sizes -12288 and 524287 bytes for example) and long messages (take 1048576 bytes for example) with non-power-of-two processes involved. Second, we fix the number of processes to 129 and then evaluate the bandwidth performance of MPI_Bcast_native and MPI_Bcast_opt by increasing message sizes from 12288 (medium message size) to 2560000 (long message size) bytes contiguously.

Figure 7 shows the throughput speedups of MPI_Bcast_opt over MPI_Bcast_native. Specifically, the significantly higher speedups are achieved for message size of 12288 bytes than for the other two cases - message sizes of 524287 bytes and 1048576 bytes. We can see that MPI_Bcast_opt performs more than two times better than MPI_Bcast_native for 12288 bytes in the case of 9,17 and 33 processes. However, as can be noted from the speedup trend in Figure 7, the speedup goes down suddenly as the transferred message sizes are increased on up to 65 processes for 12288 bytes. Regarding the case of 524287 bytes and 1048576 bytes, they show similar speedups on the measured number of processes $-9,17,33$ and 129 . In addition,

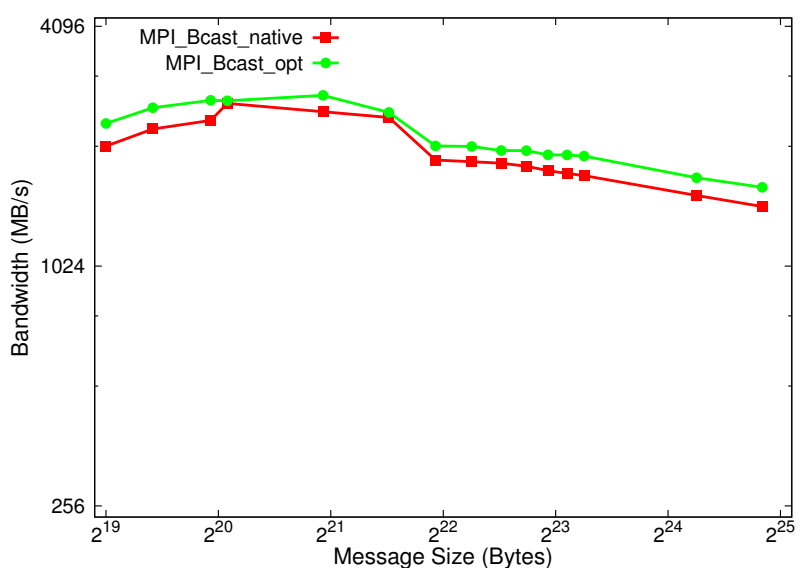

(a) $\mathrm{np}=16$

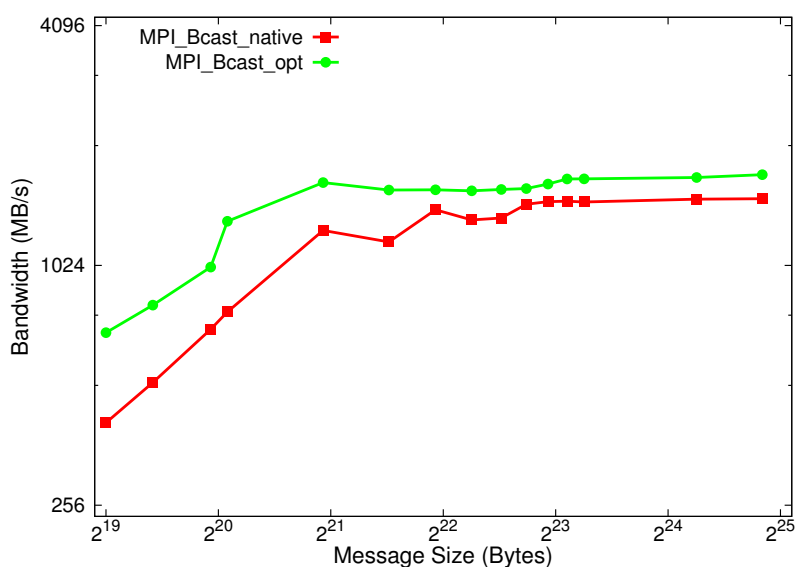

(b) $\mathrm{np}=64$

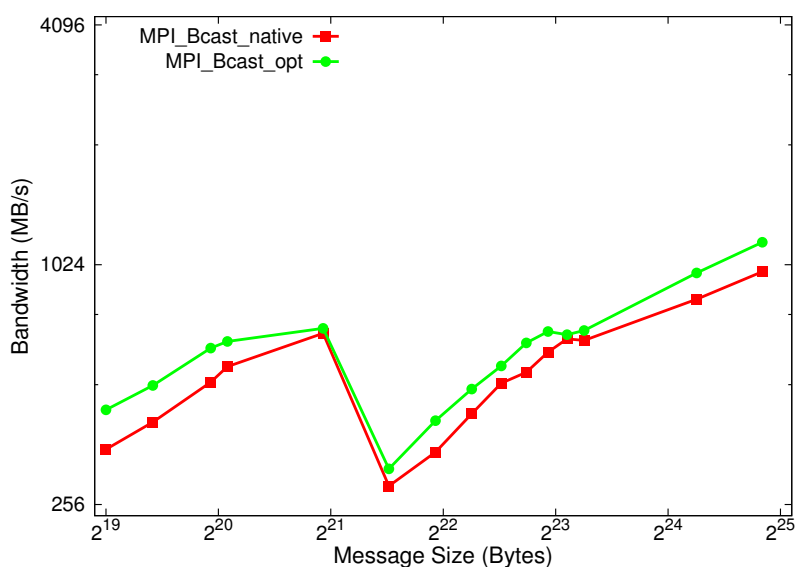

(c) $\mathrm{np}=256$

Fig. 6. Bandwidth comparison for long messages with power-of-two processes on Hornet 


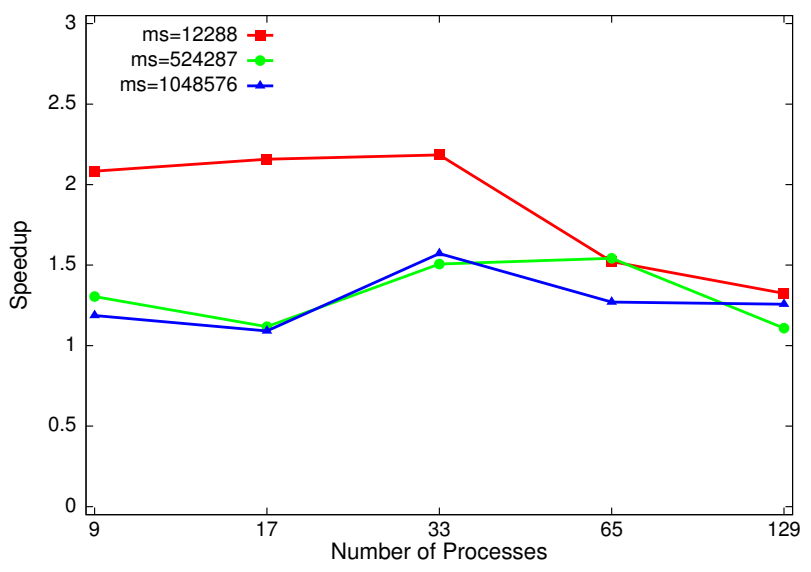

Fig. 7. Throughput speedups of MPI_Bcast_opt over MPI_Bcast_native

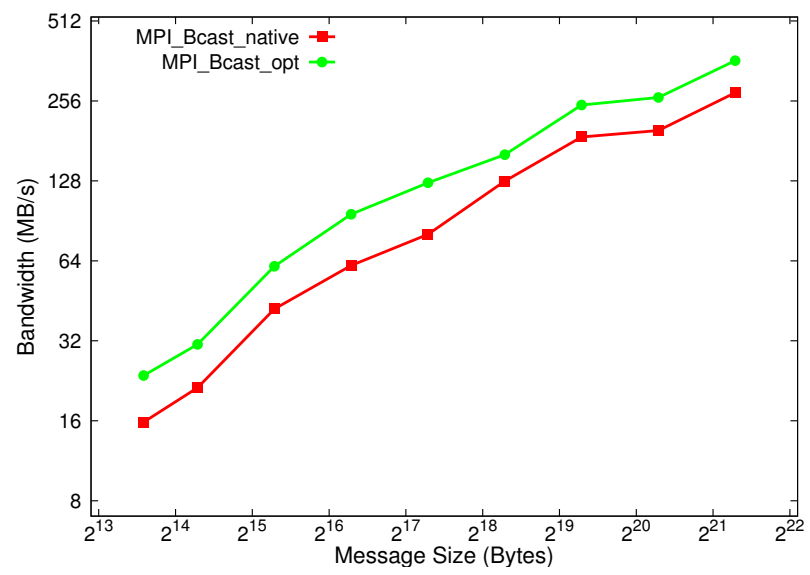

Fig. 8. Bandwidth comparison for medium and long messages with 129 processes on Hornet

compared with the case of 12288 bytes and 524287 bytes, a more stable speedup curve is presented for 1048576 bytes. In overall, we can observe that in the case of non-power-oftwo processes the MPI_Bcast_opt consistently performs better than MPI_Bcast_native in throughput no matter what ratio of inter-node messages to intra-node messages involved in the broadcast operation is.

Figure 8 takes, 129 processes for example, and shows the variance in bandwidth as the message sizes are increased from 12288 to 2560000 bytes. From this figure, we can conclude that the bandwidth increases steadily as the growth of message sizes under conditions that have sufficient memory capacity. In the best case, the bandwidth achieved by MPI_Bcast_opt get improved by up to $30 \%$ as comparison to MPI_Bcast_ori. No sudden change is expected in the curves shown in Fig. 8 since Cray MPI keeps using rendezvous message protocol for message sizes ranging from 12288 to 2560000 bytes.

\section{CONCLUSION}

In this paper, we have proposed an optimized design for the MPI broadcast collective operation based on the native broadcast algorithm used by MPICH3. We observe that, in the case of long messages or medium messages but nonpower-of-two process counts, the suboptimal ring allgather algorithm used by broadcast operation in MPICH3 leads to the inefficiency of broadcast operation. Therefore, we designed a tuned ring allgather algorithm to serve MPI broadcast implementation. Our design aims to reduce the amount of data transmission traffic brought by the native ring allgather operation, which in turn eases the burden of network and host processing. Additionally, our performance evaluation at the user level on current Cray system reveals that the tuned broadcast algorithm can reduce the bandwidth by a range from $2 \%$ to $41 \%$ for long messages with power-of-two processes (16, 64 and 256 processes). For non-power-of-two processes, the tuned design is also superior to the native one according to the throughput speedup curve. Additionally, with 129 running processes, the bandwidth achieved by the tuned broadcast design get improved by $30 \%$ at best.

\section{ACKNOWLEDGMENT}

This work has been supported by the CRESTA project funded by the European Community's Seventh Framework Programme (ICT-2011.9.13) under Grant Agreement no.287703. We gratefully acknowledge funding by the German Research Foundation (DFG) through the German Priority Programme 1648 Software for Exascale Computing (SPPEXA).

\section{REFERENCES}

[1] "The Message Passing Interface (MPI) standard," [online], http://www.mcs.anl.gov/research/projects/mpi/.

[2] MPI Forum, "MPI: A Message-Passing Interface Standard. Version 3.0," September 21st 2012, available at: http://www.mpi-forum.org/docs/ mpi-3.0/mpi30-report.pdf (Sept. 2012).

[3] A. Petitet, R. Whaley, J. Dongarra, and A. Cleary, "HPL A Portable Implementation of the High-Performance Linpack Benchmark for Distributed-Memory Computers," [online], http://www.netlib.org/benchmark/hpl/.

[4] G. Shainer, T. Liu, P. Lui, and D. Field, "The Effect of MPI Collective Operations and MPI Collective Offloads on LS-DYNA Performance," in 8th European LS-DYNA Users Conference, May 2011.

[5] "MPICH Overview," [online], http://www.mpich.org/about/overview/.

[6] "Top500 - list statistics - november 2014," http://www.top500.org/ statistics/list/ accessed: June 2015.

[7] L. Chai, Q. Gao, and D. K. Panda, "Understanding the Impact of MultiCore Architecture in Cluster Computing: A Case Study with Intel DualCore System." in CCGRID. IEEE Computer Society, 2007, pp. 471478.

[8] B. Tu, M. Zou, J. Zhan, X. Zhao, and J. F. 0002, "Multi-core aware optimization for MPI collectives." in CLUSTER. IEEE, 2008, pp. 322 325. [Online]. Available: http://ieeexplore.ieee.org/xpl/mostRecentIssue. jsp?punumber $=4655410$

[9] R. Thakur, R. Rabenseifner, and W. Gropp, "Optimization of Collective Communication Operations in MPICH." IJHPCA, vol. 19, no. 1, pp. 49-66, 2005.

[10] “MPICH Source Code," [online], http://www.mpich.org/downloads/.

[11] J. Liu, A. Mamidala, and D. Panda, "Fast and scalable MPI-level broadcast using InfiniBand's hardware multicast support," in Parallel and Distributed Processing Symposium, 2004. Proceedings. 18th International, April 2004, pp. 10-.

[12] T. Hoefler, C. Siebert, and W. Rehm, "A practically constant-time MPI Broadcast Algorithm for large-scale InfiniBand Clusters with Multicast." in IPDPS. IEEE, 2007, pp. 1-8. 
[13] K. Dashdavaa, S. Date, H. Yamanaka, E. Kawai, Y. Watashiba, K. Ichikawa, H. Abe, and S. Shimojo, "Architecture of a High-Speed MPI_Bcast Leveraging Software-Defined Network." in Euro-Par Workshops, ser. Lecture Notes in Computer Science, vol. 8374. Springer, 2013, pp. 885-894.

[14] S. Kumar, A. R. Mamidala, P. Heidelberger, D. Chen, and D. Faraj, "Optimization of MPI collective operations on the IBM Blue Gene/Q supercomputer." IJHPCA, vol. 28, no. 4, pp. 450-464, 2014. 